\title{
Restauração de dente posterior com resina composta associada à fibra de vidro: relato de caso
}

\author{
Restoring posterior teeth with composite resin \\ associated with fiberglass: a case report \\ Restauración de diente posterior con resina compuesta \\ asociada a fibra de vidrio: reporte de caso \\ Frederico dos Reis GOYATÁ \\ Fernanda de Lima VELUDO \\ Maria Fernanda Lopes FONSECA \\ Célia Regina Moreira LANZA \\ Ivan Doche BARREIROS \\ João Batista NOVAES JÚNIOR \\ Amália MORENO \\ Departamento de Clínica, Patologia e Cirurgias Odontológicas \\ Faculdade de Odontologia da Universidade Federal de Minas Gerais (FO-UFMG) 31.270-010 Belo Horizonte-MG, Brasil
}

\begin{abstract}
Resumo
A evolução das resinas compostas aliado a maior exigência estética dos pacientes, em restaurações diretas tanto em dentes posteriores como em dentes anteriores, podem ser alternativa clínica de melhor custo-benefício quando comparadas às restaurações indiretas em cerâmica. Este caso clínico tem por objetivo discorrer sobre uma técnica restauradora direta em resina composta associada ao uso de fibra de vidro para restauração de um primeiro molar superior vital com grande destruição coronária. A utilização de resina composta com fibra de vidro permitiu melhorar as propriedades mecânicas do material restaurador favorecendo a obtenção de um resultado estético e funcional satisfatório e funcionalmente eficaz em relação aos anseios do paciente e com ótima relação custo-benefício.

Descritores: Resinas Compostas; Dentística Operatória; Dente Molar; Vidro.
\end{abstract}

\begin{abstract}
The evolution of composite resins, along with greater esthetic demands by patients, in both posterior and anterior teeth, may offer a better cost-effective clinical alternative when compared to indirect ceramic restorations. This clinical case discusses a direct restorative technique in composite resin associated with the use of fiberglass to restore a vital first maxillary molar with extensive coronary destruction. The use of composite resin with fiberglass improved the mechanical properties of the restorative material favoring a satisfactory aesthetic and functionally effective result with respect to the patient's wishes while presenting an excellent cost-benefit ratio.

Descriptors: Composite Resins; Dentistry, Operative; Molar; Glass.
\end{abstract}

\section{Resumen}

La evolución de las resinas compuestas unida a la gran exigencia estética de los pacientes en restauraciones directas, tanto en dentes posteriores como en dientes anteriores, pueden ser una alternativa clinica de mejor costo-beneficio cuando comparadas a las restauraciones indirectas de cerámica. El presente caso clínico tiene por objetivo abordar aspectos sobre una técnica restauradora directa en resina compuesta asociada al uso de fibra de vidrio para restaurar un primer molar superior vital con gran destrucción coronal. La utilización de resina compuesta con la fibra de vidrio permitió mejorar las propriedades mecánicas del material restaurador favoreciendo la obtención de un resultado estético y funcional satisfactório. Siendo funcionalmente eficiente en relación a las expectativas del paciente y con excelente relación costo-benefício.

Descriptores: Resinas Compuestas; Operatoria Dental; Diente Molar; Vidrio.

\section{INTRODUÇÃO}

A estética das restaurações têm sido uma exigência dos pacientes na atualidade, manifestada pelo desejo de ter os dentes restaurados com materiais dentários, que reproduzam a forma e a cor da dentição com perfeição ${ }^{1,2}$. Dentre os procedimentos restauradores estéticos realizados pelo cirurgião-dentista na prática clínica diária, as restaurações diretas com resina composta em dentes posteriores estão cada vez mais presentes no dia a dia dos profissionais, e se destacam pelo seu excelente potencial em restabelecer a função mastigatória e a estética de forma simples e eficaz ${ }^{3-5}$.

Convém destacar que estes materiais possuem algumas limitações principalmente, com relação à extensão da cavidade e a indicação clínica, sendo que, a quantidade de estrutura coronária remanescente é fator relevante na determinação do procedimento restaurador a ser executado. Quando comparada às técnicas restauradoras indiretas com as cerâmicas reforçadas e prensadas, apresentam vantagens, tais como: preparos dentais mais conservadores, menor custo no tratamento e rapidez no procedimento restaurador ${ }^{6}$.

As restaurações diretas em cúspides funcionais que recebem carga elevada apenas com resina composta podem comprometer a longevidade do tratamento com maiores riscos de fratura e/ou falhas mecânicas. Contudo, a utilização das fibras de reforço, entre elas as fibras de vidro pré-fabricadas e impregnadas com BISGMA, associada às resinas compostas possibilitam aumento significativo na resistência flexural dos compósitos ${ }^{7-10}$, conferindo resultado mecânico adequado.

As fibras de vidro possuem propriedades desejáveis como: dureza adequada às forças mastigatórias, biocompatibilidade, adesão química à resina composta devido a presença do BISGMA, facilidade de manipulação e reforço estrutural. Tais características aliadas às propriedades da resina, que possuem boa resistência à abrasão e a compressão bem como baixa contração de polimerização, além da ampla diversidade estética, 
resultam em alternativa clínica de fácil acesso com excelentes resultados estéticos e funcionais ${ }^{11}$. O caso clínico deste estudo tem por objetivo discorrer sobre uma técnica restauradora direta com resina composta associada à fibra de vidro impregnada em BISGMA em um primeiro molar superior com grande destruição coronária, enfatizando todas as etapas clínicas desde o preparo cavitário até o acabamento final da restauração.

\section{CASO CLÍNICO}

Paciente de 21 anos de idade, sexo masculino, compareceu à Clínica de Atenção Integral ao Adulto I da Faculdade de Odontologia da Universidade Federal de Minas Gerais, queixando-se de uma lesão de cárie com grande comprometimento coronário no dente 26 . Ao exame clínico e radiográfico foi observado vitalidade pulpar, sem a necessidade de tratamento endodôntico (Figura 1). Foi acordado com o paciente, a remoção total do tecido cariado e infectado, e após isso a confecção de uma restauração direta em resina composta com reforço de fibra de vidro.

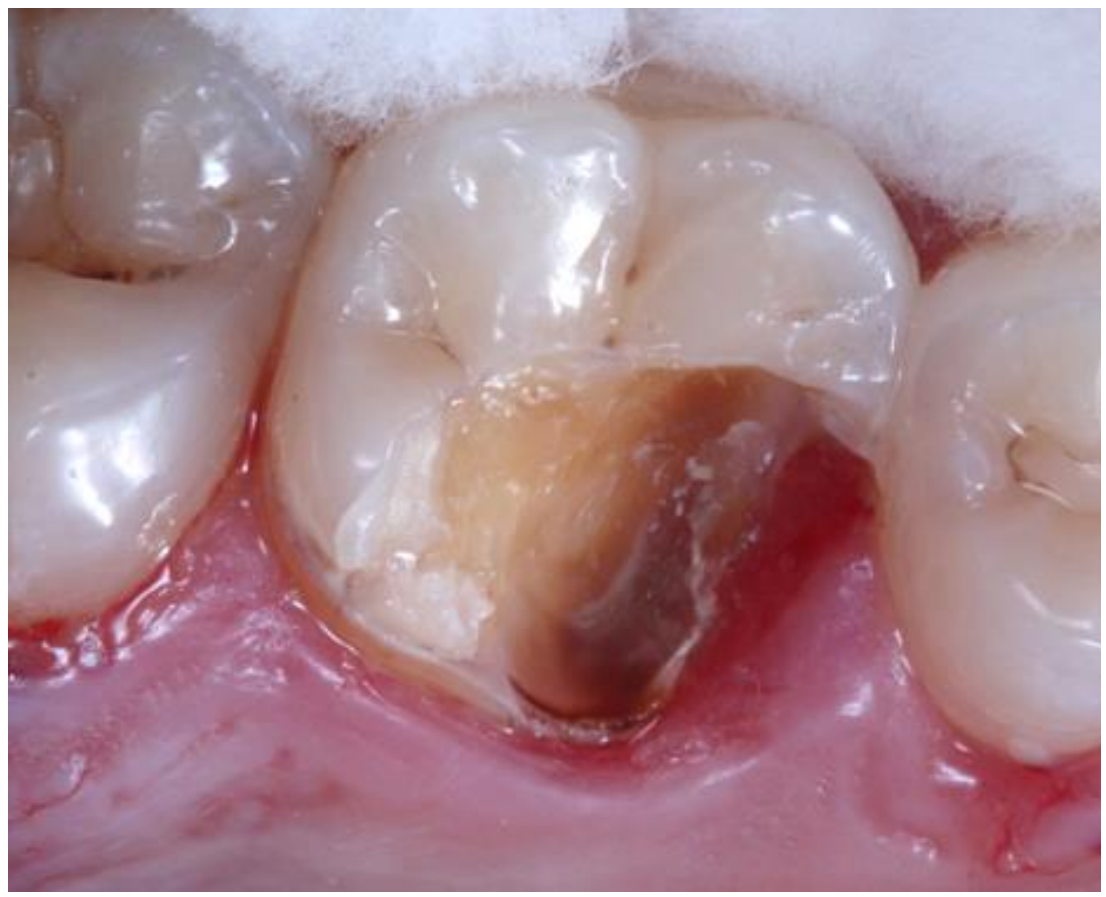

Figura 1: Aspecto clínico inicial na vista oclusal do dente 26.

Inicialmente avaliou-se os contatos oclusais com carbono AccuFilm II (Parkell, EUA), em máxima intercuspidação habitual $(\mathrm{MIH})$, profilaxia com pedra pomes e água, e seleção da cor. A seguir, procedeu-se ao isolamento relativo do campo operatório com fio afastador de algodão \#00 seco (Ultrapack-Ultradent, EUA), roletes de algodão e sucção com bomba à vácuo. Removeu-se o tecido cariado infectado com brocas carbides de aço em baixa rotação e limpeza da cavidade com água de cal. Realizou-se a proteção do complexo dentino-pulpar com cimento de hidróxido de cálcio (Life, Kerr, EUA) e cimento de ionômero de vidro modificado por resina (Vitremer, 3M Espe, EUA).

$\mathrm{O}$ esmalte e a dentina foram condicionados com ácido ortofosfórico a 37\% (Condac 37, FGM, Brasil) 20 segundos em esmalte, lavou-se por 40 segundos e secou-se. Aplicou-se o sistema adesivo (Optibond S, Kerr, EUA), e fotoativou-se por 40 segundos. Na sequência aplicou-se uma resina composta de alta viscosidade (Z350XT Flow, 3M Espe, EUA) e inseriu-se a fibra de vidro impregnada por BISGMA (Interlig, Angelus, Brasil) e fotoativou-se por 40 segundos (Figura 2). A resina composta (Premisa, Kerr, EUA) na cor A3 relativa à dentina foi inserida de forma incremental reproduzindo as características anatômicas do dente e fotoativada a cada camada por 10 segundos e o contato interproximal foi obtido com auxílio da colocação de matriz metálica $05 \mathrm{~mm}$ (Figura 3). Para finalizar a restauração, inseriu-se mais uma camada de resina composta na cor A2 (Premisa, Kerr, EUA) definindo a escultura final e o esmalte oclusal (Figura 4).

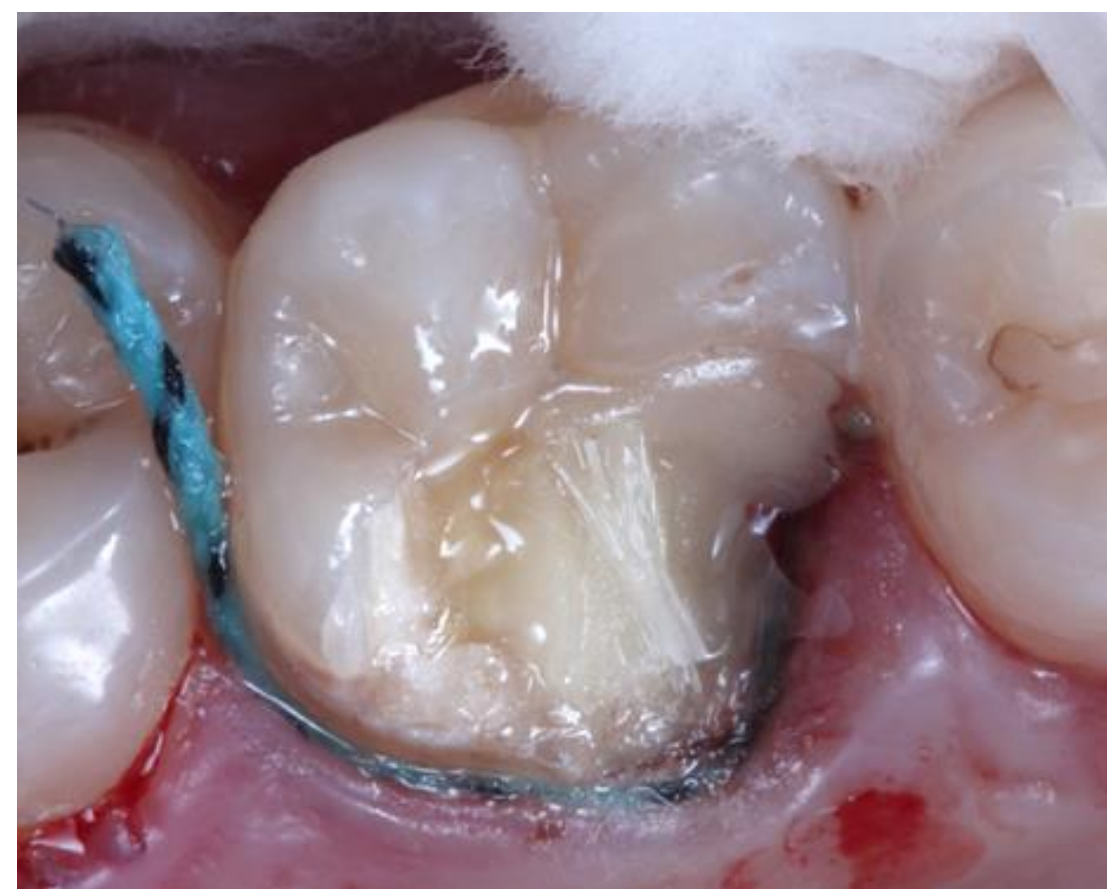

Figura 2: Resina flow e fibra de vidro inseridas na cavidade dental.

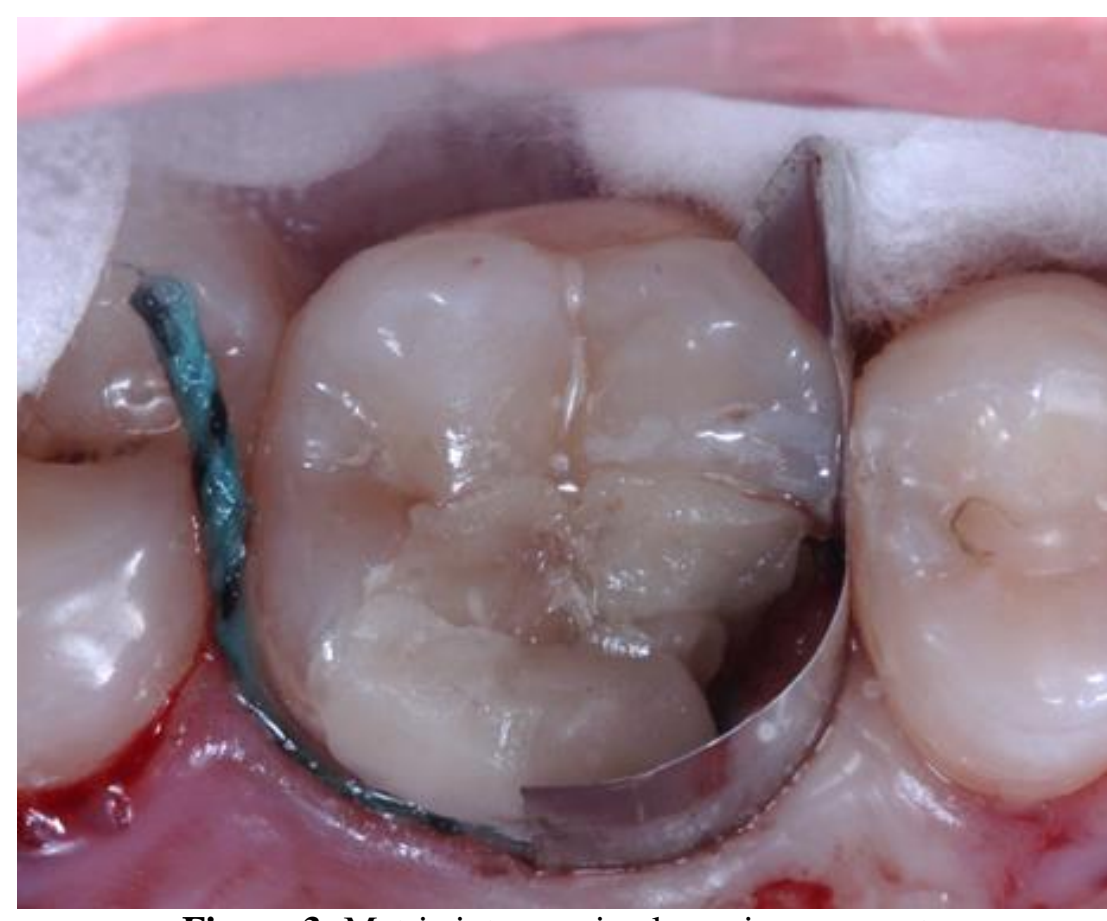

Figura 3: Matriz interproximal e resina composta na cor relativa à dentina.

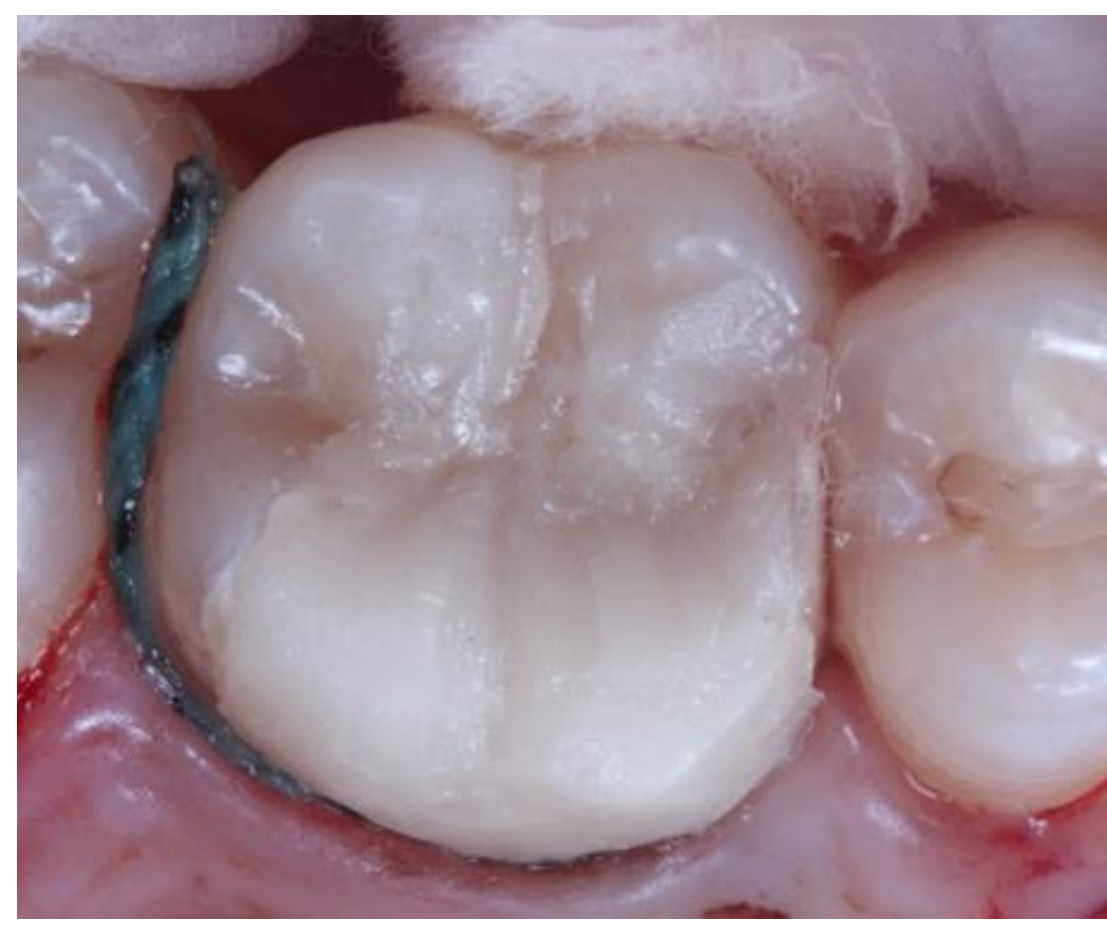

Figura 4: Aspecto clínico da restauração após sucessivas inserções incrementais de resina composta e fotopolimerização.

Realizou-se o acabamento inicial da restauração com ponta diamantada em alta rotação $2135 \mathrm{~F}$ e $2135 \mathrm{FF}$ (KG Sorensen, Brasil). Removeu-se o isolamento e procedeu-se 
a observação e ajuste dos contatos oclusais e posterior acabamento final com borrachas abrasivas (Sistema Jiffy Points, Ultradent, EUA) (Figura 5). Decorridos sete dias, realizou-se o polimento final com escova de carbeto de silício (Jiffy Brush, Ultradent, EUA) e pasta diamantada com disco de feltro (Figura 6).

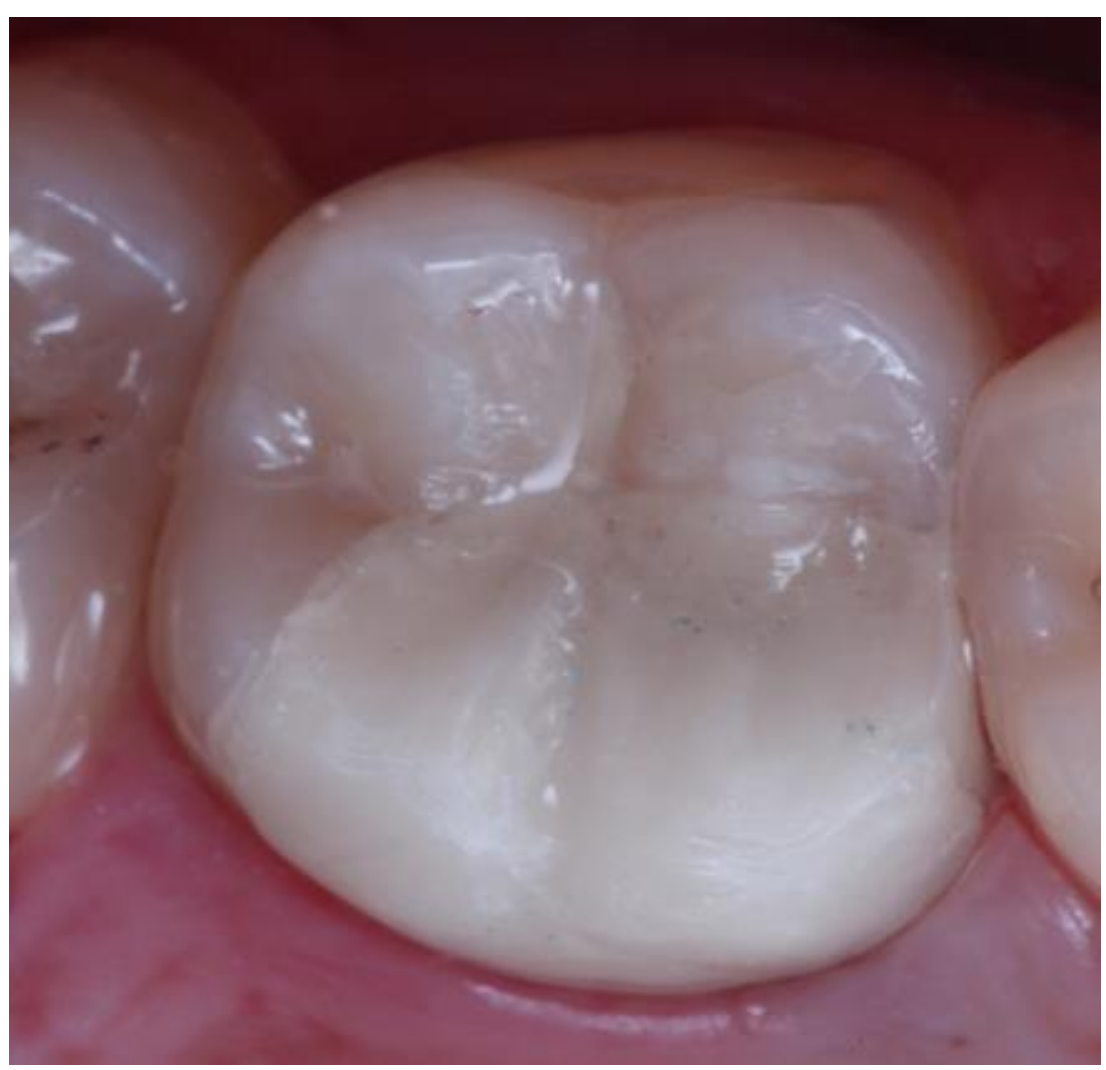

Figura 5: Aspecto clínico da restauração do dente 26.

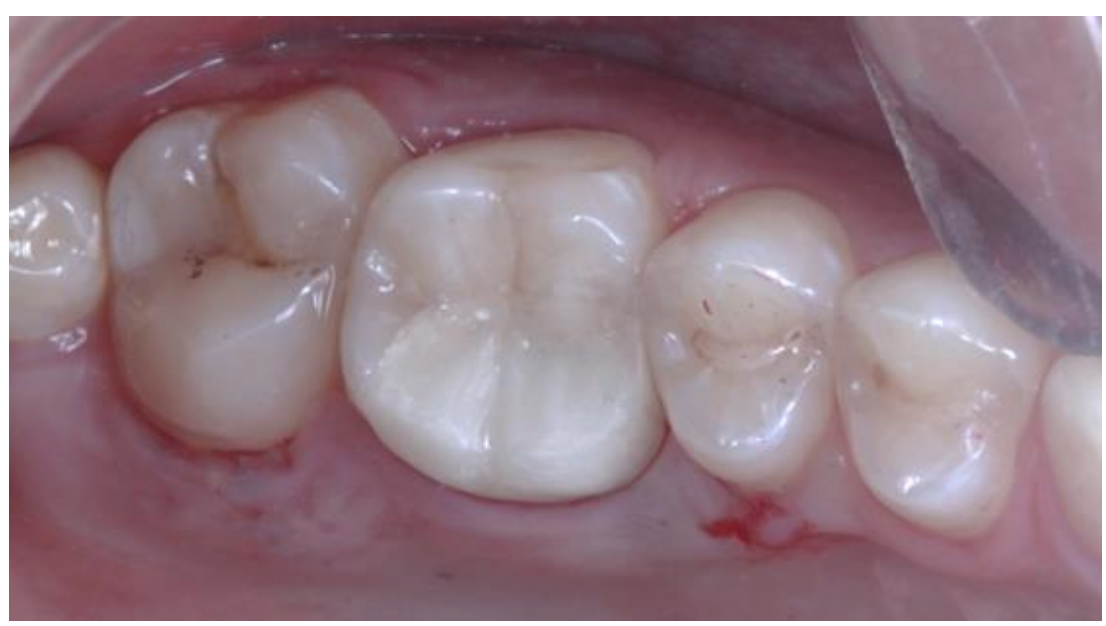

Figura 6: Aspecto clínico da restauração do dente 26 após acabamento e polimento.

\section{DISCUSSÃO}

O crescente anseio estético da população e a maior divulgação dos procedimentos e técnicas restauradoras, que tem por objetivo a recuperação da forma e função dental, bem como a exigência estética tem contribuído de fato para o desenvolvimento e o aprimoramento de novos materiais. Restaurações diretas em resina composta são procedimentos de execução relativamente fácil e de baixo custo tanto para o profissional quanto para os pacientes e proporcionam resultados clínicos imediatos, com mínima intervenção operatória, conforme demonstrado neste relato de caso ${ }^{12-13}$.

É importante observar que as restaurações diretas com resinas compostas em dentes posteriores devem seguir um protocolo de tratamento criterioso, observando-se as limitações do material e sua indicação clínica, bem como as características dos preparos cavitários e o padrão de higiene oral dos pacientes ${ }^{14}$

Fatores relacionados à técnica de inserção das resinas compostas, a espessura das porções incrementais e as diversas possibilidades e métodos de fotoativação reforçam os cuidados que os profissionais devem incluir no seu protocolo clínico-restaurador a fim de obter bom prognóstico no tratamento e longevidade clínica das restaurações $^{7-10}$, demonstrado neste trabalho com uma criteriosa inserção da resina composta de forma incremental com o principal objetivo de diminuir a contração de polimerização do material.

É conhecido que existe grande variedade de técnicas e possibilidades restauradoras descritas na literatura para a utilização das resinas compostas em dentes posteriores ${ }^{11-13}$. No entanto, é de consenso que a aplicação da resina composta deve seguir uma técnica incremental de inserção com controle rigoroso na espessura das camadas e no método de fotoativação do material restaurador ${ }^{14}$. Neste trabalho foi apresentada uma alternativa restauradora para dentes com grande destruição coronária, utilizando-se a resina composta com reforço estrutural na região de cúspide com fibra de vidro impregnada por BISGMA. Esta técnica possibilitou uma resolução simples e imediata em detrimento a um procedimento restaurador indireto em cerâmica ${ }^{15-18}$. Esta técnica tornou o tratamento menos oneroso para o paciente e constitui-se alternativa clínica viável.

Desse modo, observar os avanços dos materiais restauradores resinosos e das técnicas adesivas e se fundamentar em um planejamento restaurador criterioso são preceitos fundamentais para o sucesso com restaurações diretas em resina composta. Realizar o acompanhamento clínico periódico dos pacientes também contribui para o aumento na vida útil das restaurações.

\section{CONCLUSÃO}

A utilização das resinas compostas em dentes posteriores constitui-se alternativa clínica simples e eficaz restabelecendo a função mastigatória e estética ao paciente. A possibilidade de associar as fibras de vidro para reforço estrutural da restauração permite conferir maior resistência mecânica ao tratamento e consequentemente maior longevidade.

\section{REFERÊNCIAS}

1. Araújo EM. Cor e Forma: Elementos essenciais na estética dental. Clín int j braz dent. 2007; 2(3):108-23

2. Araujo E. Tratamento estético posterior. Clín int $\mathrm{j}$ braz dent. 2007; 3(4):334-47.

3. Aimi E, Lopes GC. Restauraçõs diretas de resina composta em dentes posteriores: uma realidade no Brasil do século XXI. Clín int j braz dent. 2007; 3(1):32-40.

4. Clavijo VR, Clavijo EMA, Andrade MF. Resina composta direta em dentes posteriores:simplificando seu uso. Dent Sci. 2008; 2(7):215-24.

5. Goyatá FR, Gilson JGR, Souza MCA, Rodrigues CRT. Restauração com resina composta microhíbrida em dente posterior - relato de caso clínico. Dent Sci. 2009; 3(9):59-66.

6. Goyatá FR, Galvão YFS, de Landa FV, Coelho LGC. Reabilitação Funcional e Estética com Fragmentos Cerâmicos em Dentes Fraturados. Clín int j braz dent. 2012; 8(4):422-29.

7. Monaco C, Ferrari M, Micelli GP, Scotti R. Clinical evaluation of fiber-reinforced composite inlay FPDs. Int J Prosthodont. 2003; 16(3):319-25.

8. Vallittu PK. Flexural properties of acrylic resin polymers reinforced with unidirectional and woven glass fibers. J Prosthet Dent. 1999; 81(3):318-26.

9. Vallitu PK, Sevelius C. Resin bonded glass fiber reinforced composite fixed partial dentures: a clinical study. J Prosthet Dent. 2000; 84(4):413-8.

Arch Health Invest 6(9) 2017 
10. Xu HHK. Whisker-reinforced heat-cures dental resin composites: effects of filler level heat-cure temperature and time. J Dent Res. 2000; 79(6):1392-7.

11. Frielich MA, Meiers JC, Duncan JP, Goldberg AJ. Fiber reinforced composites in clinical Dentistry. Hong Kong: Quintessence Books; 2000.

12. Silva LM, Francicni PAS, Veronezi MC, Nahsan FPS, Francischone LF, Sampaio PCP et al. Restauração estética em dente posterior: relato de caso clínico. $\mathrm{R}$ Dent Press Estet. 2009; 6(2):116-25.

13. Goyatá FR, Gilson JG, Cunha LG, Manta GF. Resina Composta e Matriz Oclusal - Uma Alternativa para a Restauração de Cáries Ocultas - Relato de Caso Clínico. Clín int j braz dente. 2010; 6(4):418-24.

14. Kunihira TS, Pereira-Gonçalves T, Vieira de Landa F, Guevara-Canales JO, Goulart Cruz F, Goyatá FR. Restauraciones Posteriores con Resina Compuesta - A Case Report. Kiru. 2014; 11(2):175-9.

15. Mc Laren EA, Giordano RA. Zirconia-based ceramics: Material properties, esthetics, and layering techniques of new veneering porcelain, VM9. Quintessence Dent Technol. 2005;28:99-111.

16. Griggs JA. Recent advances in materials for allceramic restorations. Dent Clin North Am. 2007; 51(3):713-27, viii.

17. Manicone PF, Rossi Iommetti P, Raffaelli L. An overview of Zirconia ceramics: Basic properties and clinical applications. J Dent. 2007; 35(11):819-26.

18. Fradeani M, Redemagni M. An 11-year clinical evaluation of leucite reinforced glass-ceramic crowns: a retrospective study. Quintessence Int. 2002; 33(7):503-10.

\section{CONFLITO DE INTERESSES}

Os autores declaram não haver conflitos de interesse.

\section{AUTOR PARA CORRESPONDÊNCIA}

\section{Amália Moreno}

amalia_moreno@yahoo.com.br

Submetido em 04/07/2017

Aceito em 10/08/2017 